Investigation of relationship between interfacial electroadhesive force and surface texture

This content has been downloaded from IOPscience. Please scroll down to see the full text.

2016 J. Phys. D: Appl. Phys. 49035303

(http://iopscience.iop.org/0022-3727/49/3/035303)

View the table of contents for this issue, or go to the journal homepage for more

Download details:

IP Address: 158.125.80.220

This content was downloaded on 03/02/2016 at 09:19

Please note that terms and conditions apply. 


\title{
Investigation of relationship between interfacial electroadhesive force and surface texture
}

\author{
J Guo, M Tailor, T Bamber, M Chamberlain, L Justham and M Jackson \\ EPSRC Centre for Innovative Manufacturing in Intelligent Automation, Wolfson School of Mechanical \\ and Manufacturing Engineering, Loughborough University, Loughborough, UK \\ E-mail: J.Guo@lboro.ac.uk
}

Received 20 July 2015, revised 18 September 2015

Accepted for publication 23 October 2015

Published 9 December 2015

\begin{abstract}
A novel investigation into the relationship between the obtainable interfacial electroadhesive forces and different surface textures is presented in this paper. Different surface textures were generated then characterized based on a recognized areal-based non-contact surface texture measurement platform and procedure. An advanced electroadhesive force measurement platform and procedure were then implemented to measure the obtainable electroadhesive forces on those different surface textures. The results show that the obtained interfacial electroadhesive forces increase with decreasing $S q$ (root mean square height) value of the substrate surface provided that the difference in $S q$ between the different substrates is over $5 \mu \mathrm{m}$. Also, the higher the applied voltage, the larger the relative increase in electroadhesive forces observed. However, when the difference of $S q$ value between different substrate surfaces is below $2 \mu \mathrm{m}$, the obtained interfacial electroadhesive forces do not necessarily increase with decreasing $S q$. Furthermore, the obtainable electroadhesive forces are not necessarily the same when the $S q$ value of two substrate surfaces are the same due to the fact that the direction of the surface texture plays an important role in achieving electroadhesive forces.
\end{abstract}

Keywords: electroadhesion, surface texture, correlation

(Some figures may appear in colour only in the online journal)

\section{Introduction}

Electrostatic adhesion, or electroadhesion [1], is an electrostatic attractive effect between two objects, i.e. the electroadhesive pad or electroadhesor and the substrate to which the pad is to be attached onto, when subjected to strong electrical fields (usually in $\mathrm{kV} \mathrm{mm}^{-1}$ range). It has been extensively used as fixtures to hold work-pieces [2], an adhesive method for space missions [3], electrostatic chucks for material handling and grasping in semiconductor industries [4], end effectors for gripping advanced composite and fibrous materials such as cloth [5] and carbon

\section{(c) (i)}

Original content from this work may be used under the terms of the Creative Commons Attribution 3.0 licence. Any further distribution of this work must maintain attribution to the author(s) and the title of the work, journal citation and DOI. fibres [6, 7], an adhesion mechanism for climbing robots [8] and material handling units for manufacturing automation and warehouse automation [9], among others. This is because electroadhesion, although it may result in relatively weaker forces (e.g. $0.1 \mathrm{~N} \mathrm{~cm}^{-2}$ ), compared with other adhesion mechanisms [10], has enhanced adaptability as it adheres to most conductive and insulating materials [11] such as smooth aluminium and rough concrete surfaces. Also, it can help bring lightweight and reduced complexity of systems in terms of control and the mechanical structure as it enables electrically controllable clamping and unclamping and no pumps or motors are required. In addition, it features ultra-low energy consumption in the $\mu \mathrm{W}$ to $\mathrm{mW}$ range as a very small current in the $\mu \mathrm{A}$ range runs through the electroadhesive pad. Furthermore, it can be applied in vacuum environments as it is non-damaging to the surface. 
The electroadhesive forces generated are subject to the contact area between the electro-adhesive pad and wall surface and the polarization property or dielectric constant of the wall substrate. The contact area is directly subject to the substrate surface texture. As such, it is necessary to take the substrate surface texture into consideration. Understanding the surface textures of contacting surfaces is of great importance to understanding any contacting phenomenon, such as the electroadhesive based contacting phenomenon. Surface texture plays an important role in obtainable electroadhesive forces [11]. The seminal work considering surface roughness as an influencing factor on the obtainable electroadhesive forces between an electroadhesive pad and a wall substrate can be found in works completed by Krape [1], where a randomly scratched and gouged circular electrode plate was employed and a $44.4 \%$ decrease in shear forces, compared with a smooth substrate surface, was obtained in ambient temperature and pressure. However, no quantification of surface roughness parameters were reported and only two substrates were used. The profile parameter, arithmetic average of the roughness profile, $R a$, was employed by Téllez et al [12] to quantify different substrate materials by using a surface profilometer. However, the same substrate material with different surface textures is required to investigate the relationship between interfacial electroadhesive forces and surface textures as the substrate material itself will greatly influence the obtainable electroadhesive forces. $R a$ may not be good enough to represent the full contacting surfaces. The most recent work by Ruffatto et al [11] selected 14 different tiles with different surface textures to exhibit the improved performance of the proposed hybrid electroadhesive pad on rough surfaces. However, no distinctive conclusions were made for the relationship between obtainable electroadhesive forces with different surface textures alone. Additionally, only some of the possible surface profiles obtained by a profilometer were applied.

This paper is intended to present a novel investigation into the relationship between the obtainable interfacial electroadhesive forces and different surface textures. In order to achieve this, an advanced electroadhesive force measurement platform and procedure, together with a recognized areal-based noncontact surface texture measurement platform and procedure, were applied. This paper starts with a brief introduction to the principle of electroadhesion, before introducing the research methodology of this research and the detailed description of each research part specified in the proposed methodology.

\section{Principle of electroadhesion}

Electroadhesion is a complicated and dynamic electrostatic attraction phenomenon with 33 variables influencing the obtainable electroadhesive forces between the electroadhesive pad and the substrate. The variables influencing the electroadhesive forces, summarized from references [1-12], are demonstrated in figure 1. Apart from these 33 variables, some other variables that may influence the electroadhesive forces obtainable should be further investigated as well. For instance,

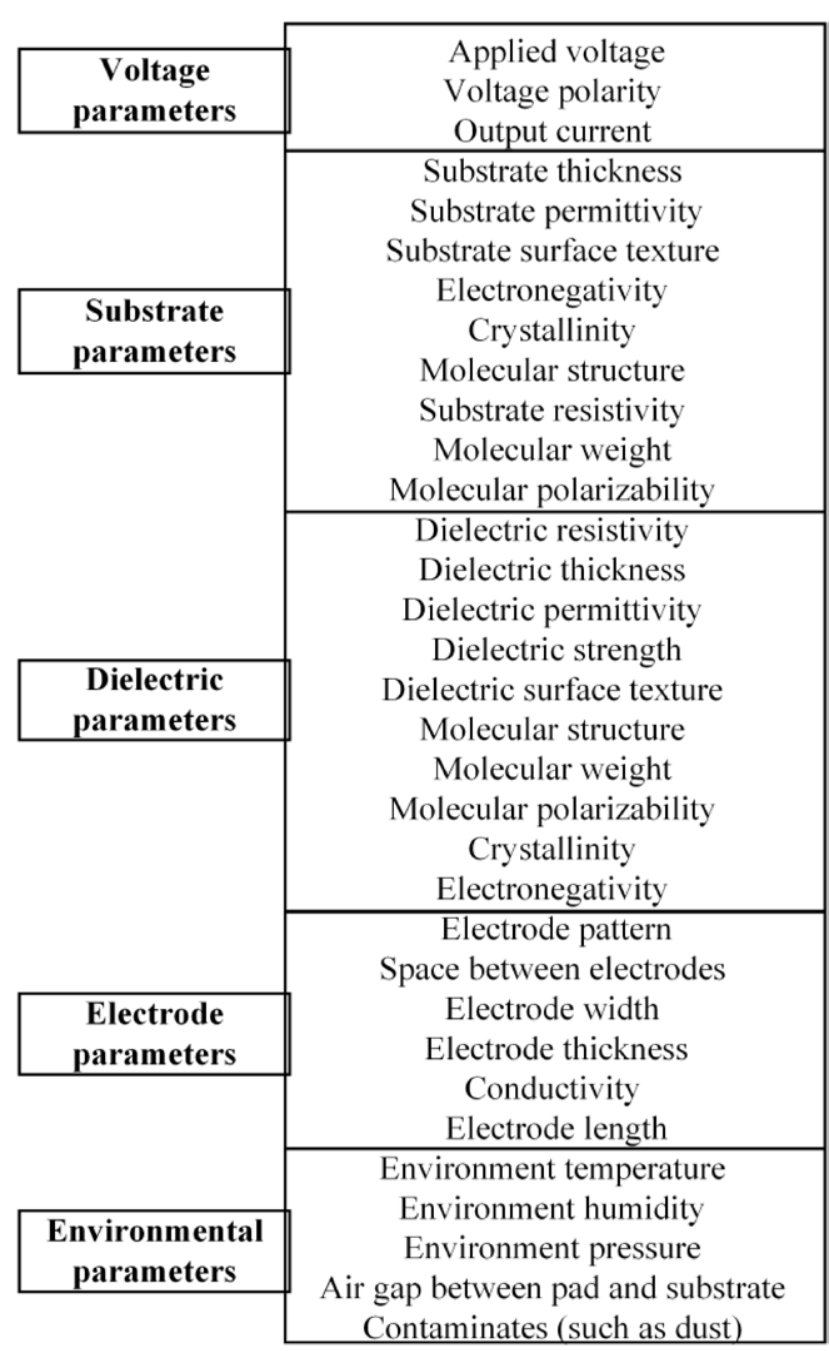

Figure 1. The major influencing parameters on electroadhesion.

if ac voltage is used, the frequency may influence the electroadhesive forces obtainable. Also, some dielectric properties such as the dissipation factor of the dielectric may influence the electroadhesive forces obtainable.

The principles of generating electroadhesive forces on conductive and insulating substrate materials are different. The former is based on electrostatic induction, whereas the latter is mainly electrical polarization [11]. A typical cross-sectional view of an electroadhesive system can be seen in figure 2, where the non-flat nature of the dielectric layer covering the electrodes and the substrate surface are presented. The modelling of the electroadhesive forces on conductive substrates can approximate to theories based on parallel capacitance whilst coplanar capacitance, together with a complicated dynamic polarization process, should be accounted for when considering insulating substrates. This paper concentrates only on using conductive and semi-conductive substrates such as aluminium plates and silicon carbide based sandpaper samples to generate the different surface textures.

For conductive substrates, Coulomb forces will be dominant if the volume resistivity of the dielectric material covering the electrodes is greater than approximately $10^{14} \Omega \mathrm{cm}$ whilst Johnsen-Rahbek forces will be dominant if the volume 


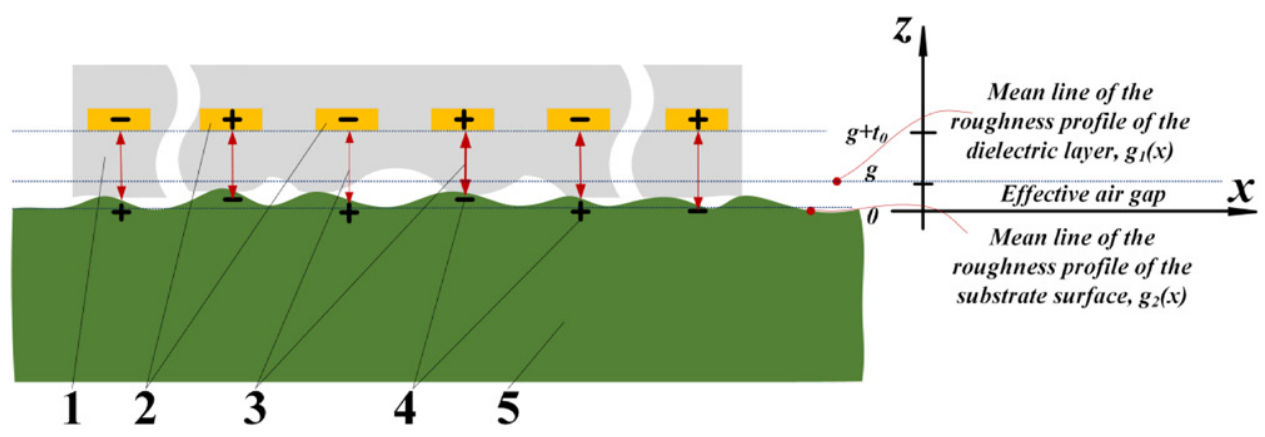

Figure 2. Cross-sectional diagram of an electroadhesive system, where 1 denotes the dielectric material, 2 denotes the electrodes connected with high voltage supplies, 3 denotes the electroadhesive forces between the electroadhesive pad and the substrate, 4 denotes the induced charges, 5 denotes the substrate to which the pad adheres onto, $t_{0}$ denotes the effective thickness of the dielectric layer and $g$ denotes the effective air gap between the pad and the substrate surface.

resistivity is between approximately $10^{10}$ and $10^{12} \Omega \mathrm{cm}[13]$. The theoretical modelling of electroadhesive forces on conductive substrates were mostly based on the Maxwell stress tensor method and the virtual work method, and is always based on several assumptions [14] such as that the dielectric material is rigid, linear, homogenous, isotropic and neutrally charged, the scale of the area of the pad is far larger than the thickness dielectrics within the capacitor and the fringing fields are neglected. Usually, the electroadhesive forces between the pad and grounded conductive substrates are different from those substrates where a permanent earth is not ensured [7]. Although the saturated electroadhesive forces obtained for conductive substrates is quick (usually within $1 \mathrm{~s}$ ), a dynamic electrostatic attraction force generation process can still be seen on conductive substrates after applying voltage on the electroadhesive pad [15]. A more comprehensive and accurate model should also take edge effects [16], surface texture information of the pad and substrate and environmental parameters into consideration.

\section{Relationship between the electroadhesive forces and surface textures}

The aim of this research is to identify the relationship between the obtainable interfacial electroadhesive forces generated on different substrate surface conditions by an electroadhesive pad and surface textures. To this end, four major stages have been addressed, forming the research methodology guiding the research reported in this paper.

The first stage of this research was to generate a range of different surface textures by sanding aluminium plates (grade 1050) and using selected sandpaper samples directly. Following this, surface texture measurements and quantification of those surfaces were conducted using Alicona InfiniteFocus and DigitalSurf MountainMap (v5) respectively. Alicona is a non-contact surface texture measurement platform based on focus variation [17] and MountainMap is a common commercial surface texture data analysis software widely used in research and industries [18]. Once the characterization of those surfaces was completed, the electroadhesive forces were measured using an advanced electroadhesive force measurement platform and procedure. Finally, the correlation between electroadhesive forces and surface textures was identified.

\subsection{Surface texture preparation and characterization}

As sandpaper samples can bring a large range of surface textures, three A4 size silicon carbide sandpaper samples were selected, with grit designations of P120, P400 and P1200. A random area of $7.86 \mathrm{~mm} \times 5.78 \mathrm{~mm}$ on each sandpaper sample was measured and the surface texture information of the selected area of the three sandpaper samples in three dimensions can be seen in figure 3, where $S q=4.8 \mu \mathrm{m}$ for $\mathrm{P} 1200, S q=10.7 \mu \mathrm{m}$ for $\mathrm{P} 400$ and $S q=31.5 \mu \mathrm{m}$ for P120. The same data analysis procedure was carried out for each sample. The raw data was uploaded into MountainMap and any non-measured data was filled in. Filtrations were then conducted, including removing the surface form/slope and waviness, before the quantification of each surface was completed using areal parameters.

Four smooth circular aluminium plates, with a thickness of $1 \mathrm{~mm}$ and a diameter of $275 \mathrm{~mm}$, were selected to further investigate the effect of the direction of the surface texture on the obtainable electroadhesive forces. One plate was kept without sanding, the other three were sanded by a 60 grit aluminium oxide sanding disc to generate different surface texture directions, uni-directional, bi-directional, and multidirectional. Ten random areas, each $1.43 \mathrm{~mm} \times 1.09 \mathrm{~mm}$ were measured from each plate. The surface texture information of a typical area of each aluminium plate can be seen in figure 4 . The average $S q$ of the 10 selected areas of each plate was $S q=1.5 \mu \mathrm{m}$ for the original plate, $S q=2.8 \mu \mathrm{m}$ for the unidirectional sanded plate, $S q=3.1 \mu \mathrm{m}$ for the bi-directional sanded plate and $S q=2.8 \mu \mathrm{m}$ for the multi-directional sanded plate. The standard deviations of the ten measured areas of the three scratched plates were all within $10 \%$. Also, for both the sandpapers and the aluminium plates, the recognized standard areal Gaussian filter and a cut-off length of $0.8 \mathrm{~mm}$ [19] were selected, and the end effects were managed.

\subsection{Electroadhesive force measurement}

3.2.1. Electroadhesive pad design and manufacturing. Since the comb or interdigitated electroadhesive pad geometry is the 


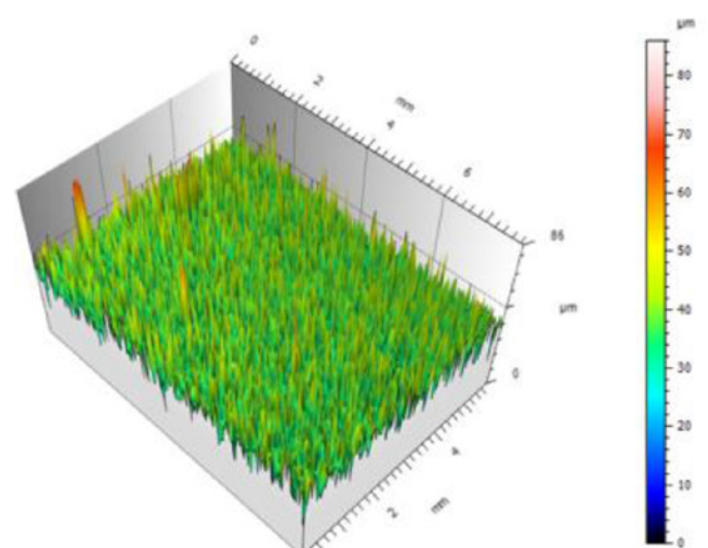

(a)

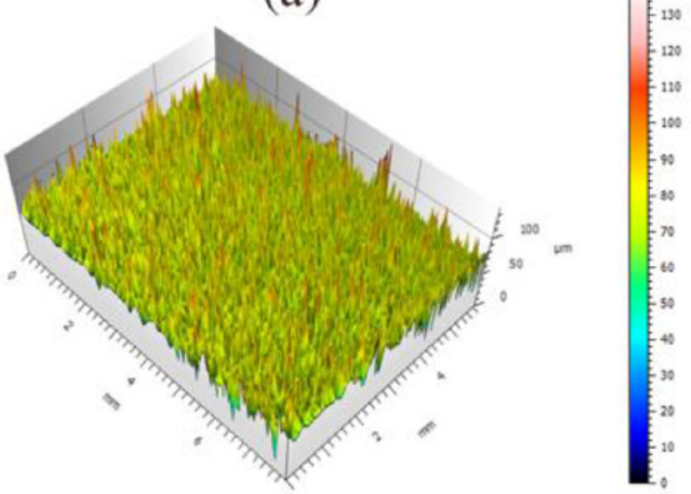

(b)

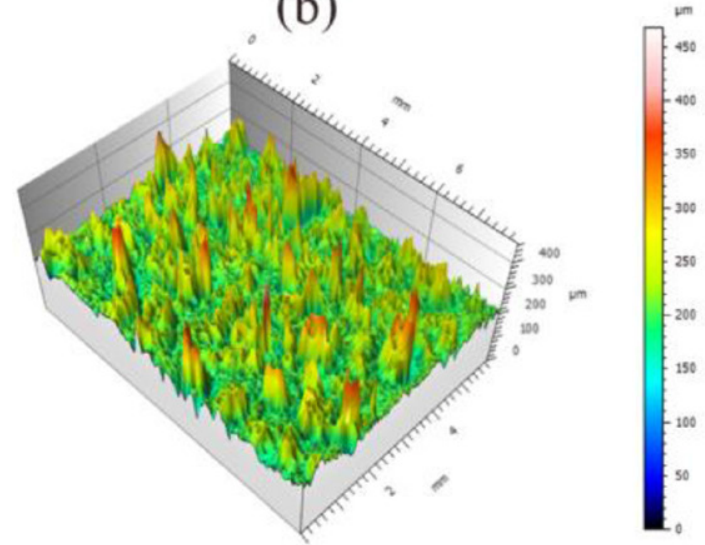

(c)

Figure 3. Surface texture information of sandpaper samples: (a) P1200; (b) P400; (c) P120.

one of the most widely used pad geometry, a comb shape pad was designed using Solidworks. It was professionally etched and coated using a polyurethane conformal spray coating. The electroadhesive pad design and manufacturing process can be seen in figure 5. The copper area outside of the interdigitated pattern was for supporting the pad flat enough during the coverlaying process. Only the interdigitated pattern part of the design was used for the electroadhesive force testing. The effective pad area is $190 \mathrm{~mm} \times 230 \mathrm{~mm}$. The space between electrodes, electrode width and thickness are $2 \mathrm{~mm}, 2 \mathrm{~mm}$ and $40 \mu \mathrm{m}$ respectively. The base dielectric material covering the electrode is a $25 \mu \mathrm{m}$ Polyimide (PI) with a $13 \mu \mathrm{m}$ Polyacrylates adhesive. Since the mentioned Alicona cannot measure the pad surface, surface texture information of a random area of the polyimide base side was measured by a Zygo newview 5000 as shown in figure 6, where a Mirau 10X was employed. The reason why Alicona could not be used to measure the PI pad surface was because that the surface of the PI used in this research was quite shiny and transparent. Plenty of missing data were found when using the Alicona to measure the PI surface. Zygo is also a non-contact surface texture measurement platform based on coherence scanning interferometry [19]. The recognized standard Gaussian filter and a cut-off length of $0.8 \mathrm{~mm}$ were applied, and the end effects were managed during the data analysis process in Talymap. The $S q$ of the polyimide base is $0.2 \mu \mathrm{m}$.

\subsubsection{Electroadhesive force measurement platform and pro-} cedure. A mechatronic and reconfigurable electroadhesive force measurement platform was used to obtain the interfacial electroadhesive forces between the pad and substrates. The system diagram can be seen in figure 7(a), where a 6-axis ATI gamma force/torque (F/T) sensor was used to record the electroadhesive forces. The communication between the F/T sensor and the computer was through a netbox via an Ethernet cable and the data was selected to be sampled at $152 \mathrm{~Hz}$. The linear rail can achieve vertical movement using a servo motor with encoder driven by a Kollmorgen motor driver connected with a CompactRio. This allows almost real time control of the linear rail via a Xilinx FPGA which is designed to communicate with the computer via Ethernet. The smallest movement of the linear rail is approximately $0.8 \mu \mathrm{m}$. The electroadhesive pad was connected with two EMCO high voltage converters with $( \pm) 0-10 \mathrm{kV}$ output and $0-5 \mathrm{~V}$ reference input. The reference input was from a direct current power supply unit, Instek GPD3303, which was designed to communicate with the computer through via a USB. The electroadhesive force measurement platform can be seen in figure 7(b). A Labview interface was developed for interactive control of the movement of the linear rail, changing the supply voltage, recording and saving the electroadhesive force data. Please note that electrical safety interlock system and safety screens were applied in this advanced measurement platform.

In order to investigate the relationship between the interfacial electroadhesive forces and surface textures, only the surface texture was varied whilst keeping all other influencing variables as constant, can be seen from table 1. Please note that as the obtainable electroadhesive forces may change during the day and between days, the experiments were conducted at constant times, from 2 to $7 \mathrm{pm}$, when less variation in room temperature, humidity and air pressure was observed, as can be seen in table 1 . For each selected surface texture, five experiments were repeated. The electroadhesive force measurement procedures can be seen in figure 8 , where the aforementioned dynamic changing of the obtainable electroadhesive forces is presented. This dynamic electrostatic attraction phenomenon can be seen in step 3, the pad charging phase after turning on the voltage, as shown in figure 8 . The pull-off velocity $\left(0.1 \mathrm{~mm} \mathrm{~s}^{-1}\right)$ and pull-off acceleration (50 revs ${ }^{-2}$ ), charge time $(90 \mathrm{~s})$ and discharge time $(510 \mathrm{~s})$ were maintained at constant values when conducting the experiments. 

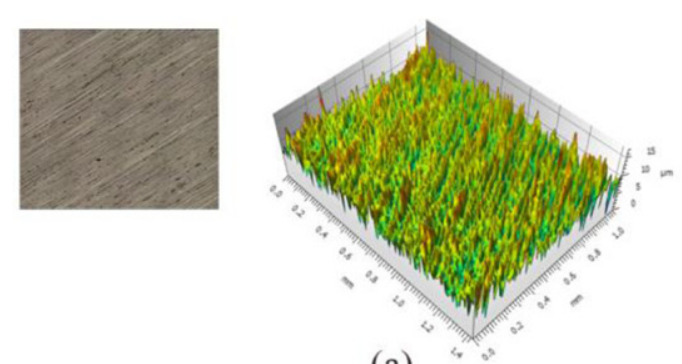

(a)
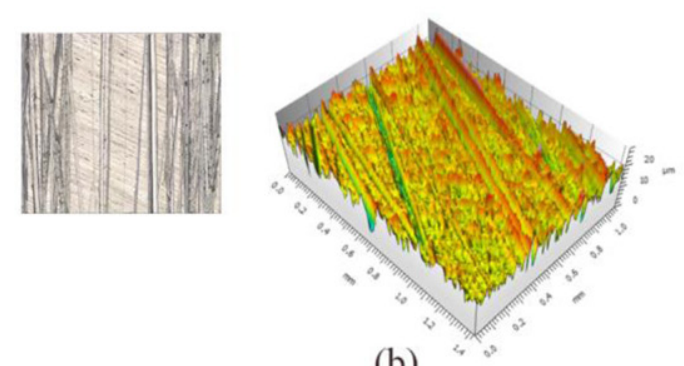

(b)
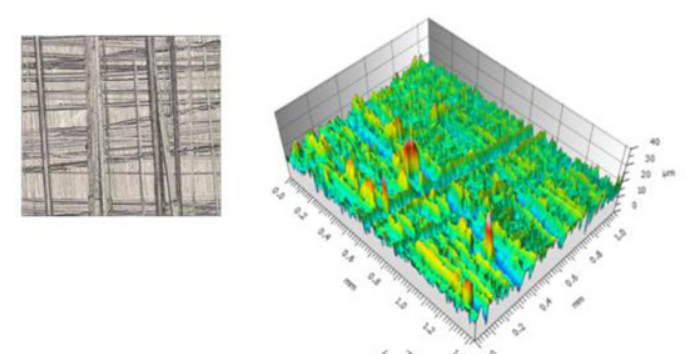

(c)
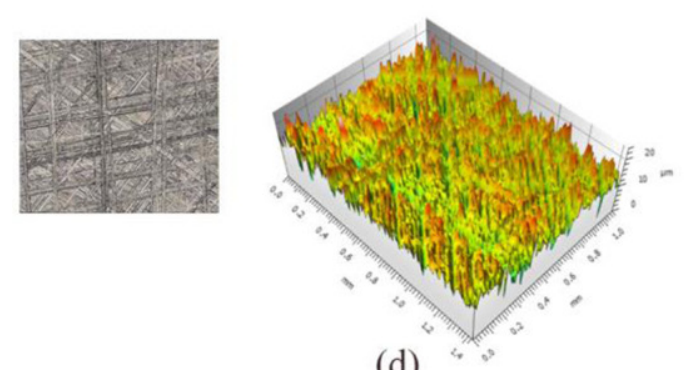

(d)

Figure 4. Surface texture information for: (a) the original plate; (b) the plate with uni-directional scratches; (c) the plate with bidirectional scratches; (d) the plate with multi-directional scratches.

During the residual charge dissipation process, the pad was grounded for $300 \mathrm{~s}$ after each test. Also, the aluminium plate with uni-directional scratches was grounded for $300 \mathrm{~s}$ each time before rotating it to a different angle to investigate the influence of surface texture directions on the electroadhesive forces obtainable. An electrostatic fieldmeter, FMX-003, was used to compare the surface charge value of the plate before applying the voltage and after the grounding. $300 \mathrm{~s}$ was enough to obtain similar results that were less than 5\% difference.

\section{Results}

The correlation between the obtainable interfacial electroadhesive forces and surface textures was performed after

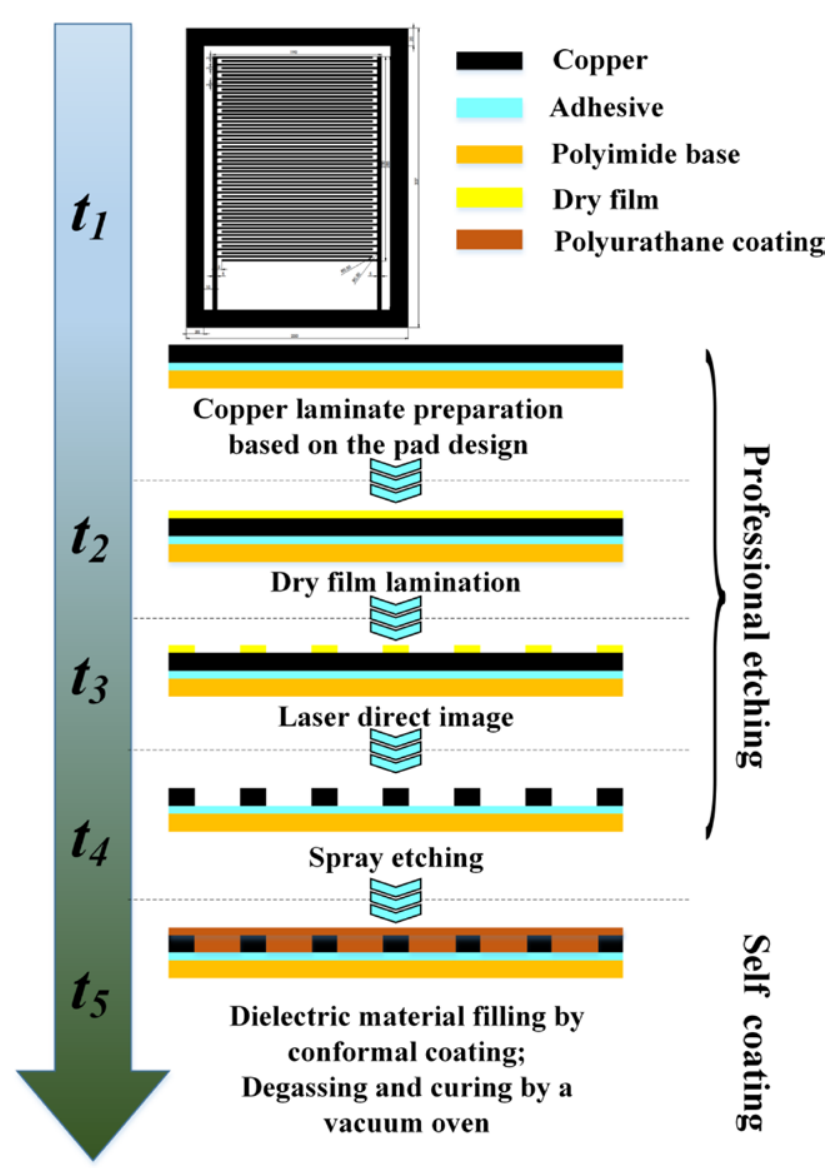

Figure 5. Electroadhesive pad design and manufacturing process.

obtaining the surface texture parameter and electroadhesive force data.

\subsection{Results on the sandpaper samples}

It can be seen from figure 9 that the obtained interfacial electroadhesive forces increase with decreasing $S q$ values on the sandpaper samples. This may be due to the fact that the larger the $S q$ value, the less the air gap and therefore more contact area between the pad and the substrate. Also, the higher the voltage applied, the larger the relative increase in the electroadhesive forces. Between the sandpaper samples P120 and P400, for instance, a relative increase of $9.1 \%$ was achieved at $2 \mathrm{kV}$, but $21.2 \%$ at $4.4 \mathrm{kV}$ and $31 \%$ at $6 \mathrm{kV}$. Please note that the stated voltage supplied was based the voltage difference between the positive electrodes and negative electrodes. The reason why $4.4 \mathrm{kV}$ was selected rather than $4 \mathrm{kV}$ was because the voltage output of the power supply unit was not stable at $4 \mathrm{kV}$. Electroadhesive force measurements on applying voltage that is greater than $6 \mathrm{kV}$ were not conducted due to possible electric discharge and dielectric breakdown of the pad.

\subsection{Results on the aluminium plates}

The electroadhesive forces obtained on the aluminium plates with various surface textures can be seen in figure 10. Based on bar B and bar D, it can be concluded that the obtainable electroadhesive forces are not necessarily the same 


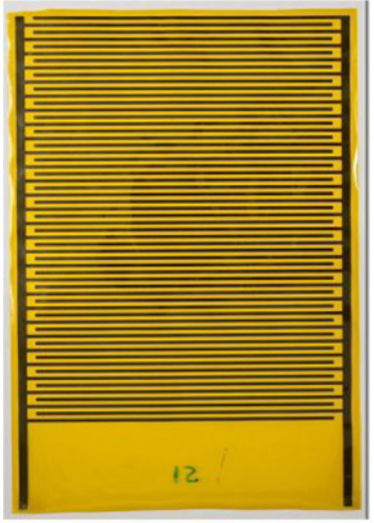

(a)

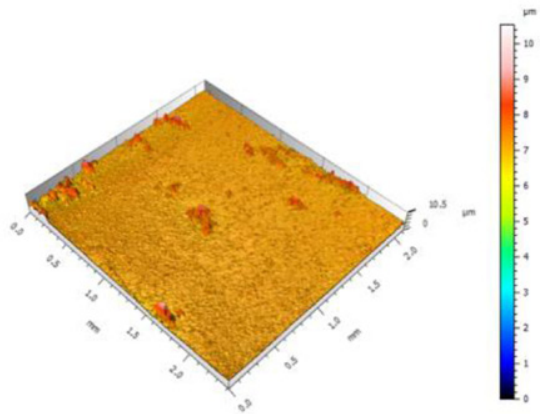

(b)

Figure 6. The pad: (a) the PI base side; (b) surface texture information of the PI base.

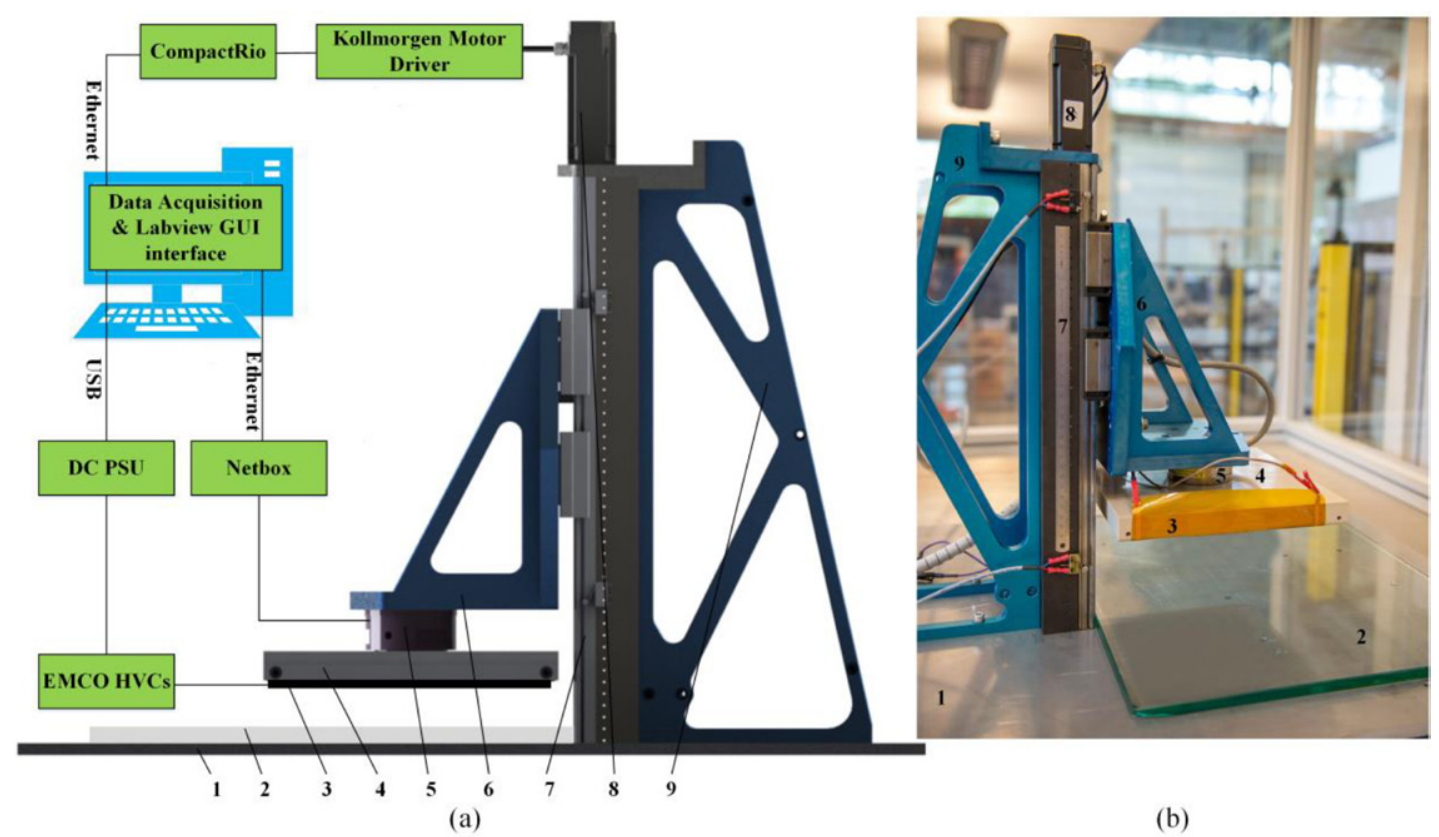

Figure 7. Electroadhesive force measurement platform: (a) system diagram, where 1 is the test rig base, 2 is the toughened glass, 3 is the pad, 4 is the pad holder, 5 is the ATI F/T sensor, 6 is the pad holder supporter, 7 is the linear rail, 8 is the servo motor and 9 is the rail supporter; (b) physical setup.

even when the $S q$ values of the two substrate surfaces are the same. This may also suggest that a single $S q$ cannot be used to represent the effective air gap between the pad and the substrate. In addition, the sanded aluminium surface with horizontal scratches has a relative increase of $38.1 \%$ more electroadhesive forces than the surface with multi-directional scratches. Based on bar A, B and C, when the difference of the $S q$ values between different substrate surfaces is within $2 \mu \mathrm{m}$, the obtained interfacial electroadhesive forces do not necessarily increase with decreasing $S q$ values as observed with the sandpaper samples. This may due to the fact that, based on the data shown from bar $\mathrm{C}$ to bar $\mathrm{G}$, the directions of surface texture on substrate surfaces play an important role in achieving electroadhesive forces. Compared with the original aluminium plate (bar C), the aluminium surface with approximately $45^{\circ}$ (bar E), $90^{\circ}$ (bar F) and $135^{\circ}$ (bar G) scratches all have obtained slightly less forces (around 10\%). The aluminium surface with approximately horizontal scratches, however, has a $59.2 \%$ increase in the obtained electroadhesive forces. Note that the bar D, E, F and G in figure 10 were based on the same plate. Different scratch orientations were obtained by rotating the plate. Also, the scratch directions relative to the electrode pattern direction was depicted on the left side in figure 10.

\section{Conclusion and future work}

The work presented in this paper has focused upon the investigation into the relationship between the obtainable interfacial electroadhesive forces and different surface textures. The key findings from this work are:

- The obtained interfacial electroadhesive forces increase with decreasing $S q$ value of the substrate surface provided 
Table 1. Controlled parameters for the electroadhesive force measurement.

\begin{tabular}{|c|c|c|}
\hline Controlled parameters & Sandpaper samples & Aluminium plates \\
\hline Applied voltage $(\mathrm{kV})$ & $2,4.4$ and 6 & 4.4 \\
\hline Polarity of applied voltage & \multicolumn{2}{|c|}{ Dual polarity } \\
\hline Substrate material and thickness & $\begin{array}{l}1 \mathrm{~mm} \text { sandpaper samples with } 12 \mathrm{~mm} \text { toughened } \\
\text { glass }\end{array}$ & $\begin{array}{l}1 \mathrm{~mm} \text { aluminium plates with } 12 \mathrm{~mm} \text { toughened } \\
\text { glass }\end{array}$ \\
\hline Substrate surface texture & \multicolumn{2}{|c|}{ Varies } \\
\hline Dielectric material and thickness & \multicolumn{2}{|c|}{$25 \mu \mathrm{m}$ polyimide and $13 \mu \mathrm{m}$ polyacrylates adhesive } \\
\hline Pad surface roughness $(S q, \mu \mathrm{m})$ & \multicolumn{2}{|c|}{0.2} \\
\hline Electrode pattern and area & \multicolumn{2}{|c|}{ Comb shape and pad area of $190 \mathrm{~mm} \times 230 \mathrm{~mm}$} \\
\hline Space between electrodes (mm) & \multicolumn{2}{|r|}{2} \\
\hline Electrode width (mm) & \multicolumn{2}{|r|}{2} \\
\hline Electrode thickness $(\mu \mathrm{m})$ & \multicolumn{2}{|c|}{40} \\
\hline Electrode material & \multicolumn{2}{|c|}{ Copper } \\
\hline Environment temperature $\left({ }^{\circ} \mathrm{C}\right)$ & \multicolumn{2}{|c|}{$21.5 \pm 0.1$} \\
\hline Environment humidity (\%) & \multicolumn{2}{|c|}{$43 \pm 1$} \\
\hline Environment pressure $(\mathrm{hPa})$ & \multicolumn{2}{|c|}{$1003 \pm 1$} \\
\hline Preload (air gap, N) & \multicolumn{2}{|c|}{$34 \pm 1$} \\
\hline
\end{tabular}

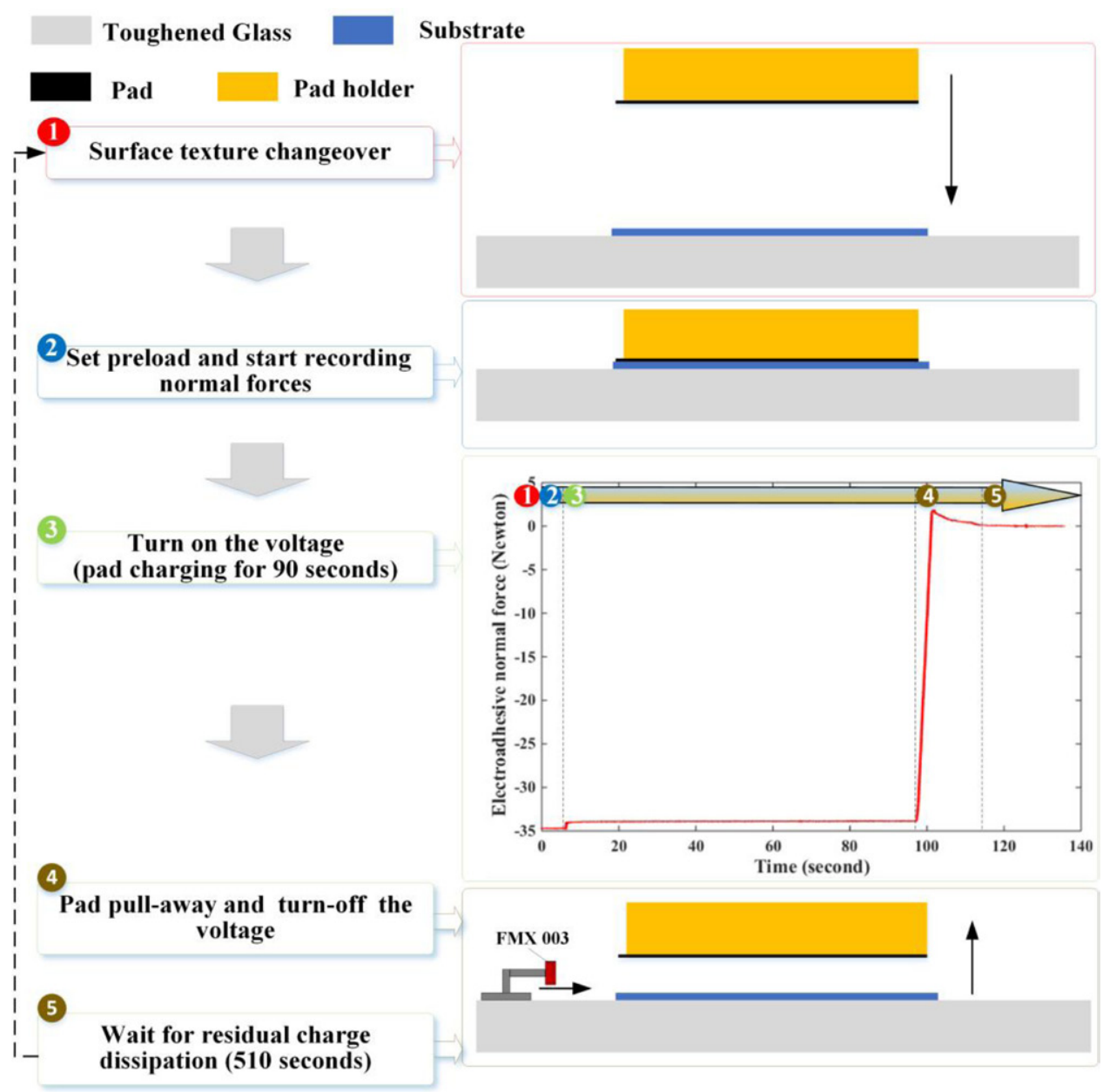

Figure 8. Electroadhesive force measurement procedures.

that the difference of $S q$ between different substrates is greater than $5 \mu \mathrm{m}$.

- The higher the applied voltage, the larger the relative increase in the obtainable electroadhesive forces.

- When the difference in $S q$ value between different substrate surfaces is within $2 \mu \mathrm{m}$, the obtained interfacial electroadhesive forces do not increase with decreasing $S q$ value.

- The obtainable electroadhesive forces are not the same when $S q$ value of two substrate surfaces are the same due to the fact that the direction of the surface texture plays an important role in achieving electroadhesive forces. 


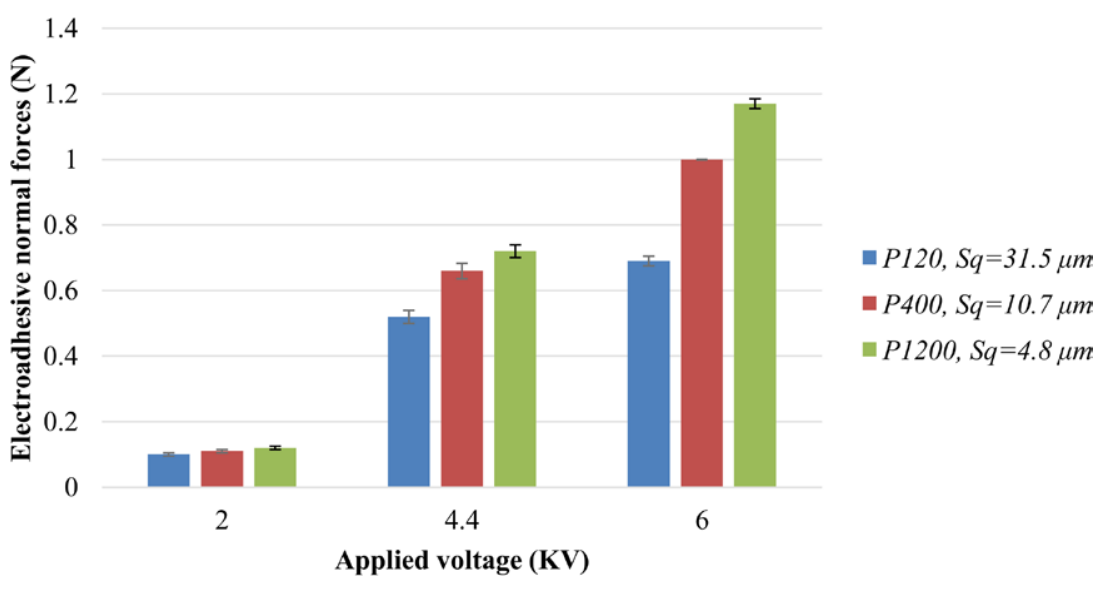

Figure 9. Electroadhesive forces on sandpaper samples.

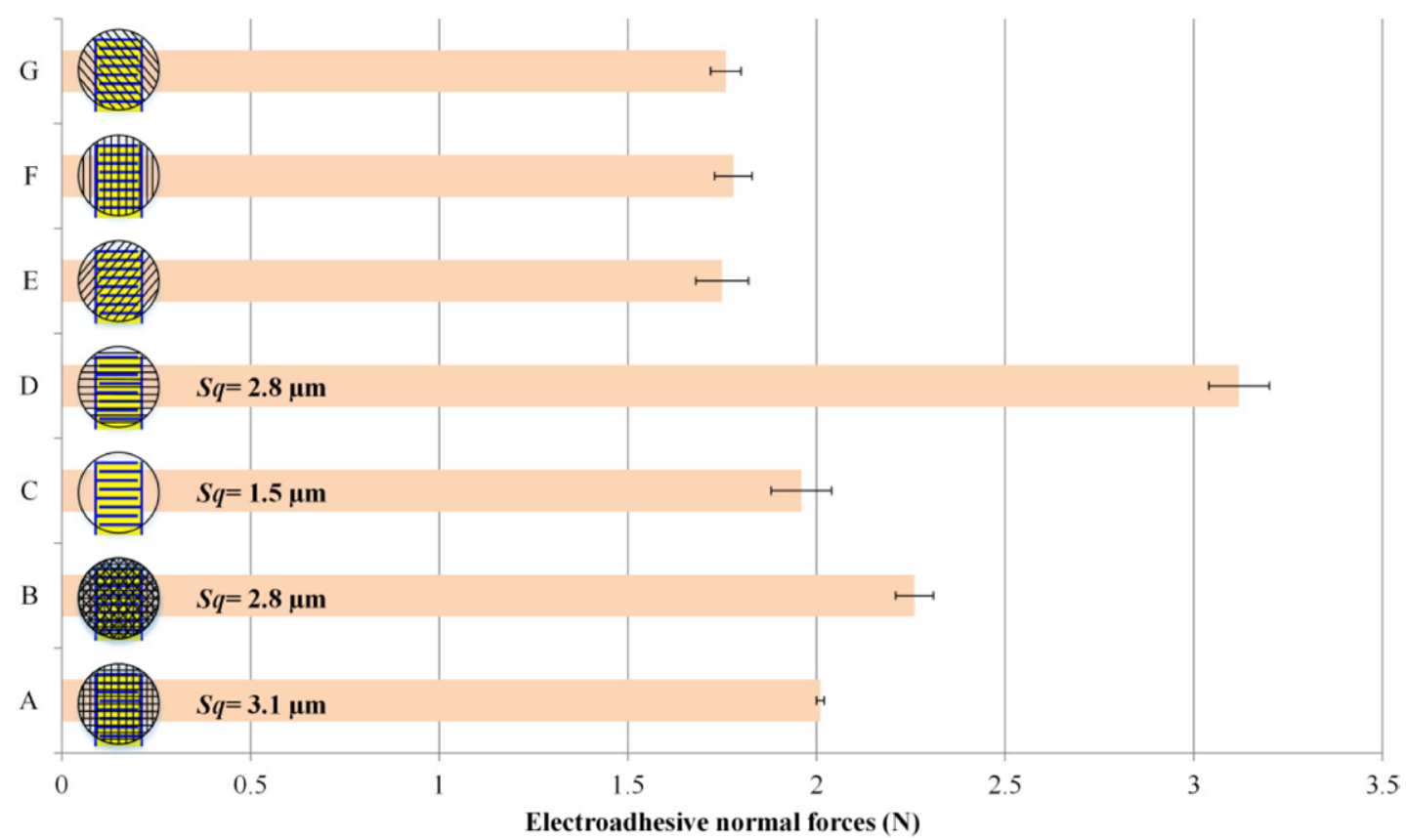

Figure 10. Electroadhesive forces on aluminium plates, where A denotes the sanded surface with bi-directional scratches, B denotes the sanded surface with multi-directional scratches, $\mathrm{C}$ denotes the original plate surface, $\mathrm{D}$ denotes the sanded surface with horizontal scratches, E denotes the sanded surface with $45^{\circ}$ scratches, F denotes the sanded surface with vertical scratches and G denotes the sanded surface with $135^{\circ}$ scratches.

Some work of interest and will be published in future has been identified as:

- Varying different surface textures of the dielectric layer facing the substrate whilst keeping the surface texture of the substrate the same to investigate the obtainable interfacial electroadhesive forces.

- Varying different pad geometries such as spiral pattern whilst maintaining the same surface texture of the dielectric layer and the substrate to investigate the relationship between the interfacial electroadhesive force and pad geometries. The spiral pattern would be independent of the scratch directions. Also, different electrode widths and spaces of the same pad geometry will be used to investigate how these two variables influence the relationship.

- Investigation of the relationship between interfacial electroadhesive forces and different structured substrate surface textures and a more accurate theoretical model considering surface roughness for industry applications.

- Investigation of using a layer of soft foam backing to the pad to conform to rough surfaces rather than using compliant dielectrics such as Vytaflex [11] and fluid dielectrics [20] and inductive fibres [21]. The Vytafelx attracts dust and hardens over several weeks of use. The film enclosing the fluid dielectrics may become damaged easily. The polymeric electrostatic inductive fibres may be difficult and expensive to manufacture.

The results showed that it is necessary to have similar surface texture of substrate surfaces when investigating the relationship between the obtainable electroadhesive forces and other influencing factors on electroadhesion such as substrate material. Also, the results presented in this paper and the outlined future work may both show that adaptive electroadhesion 
is necessary to enable the electroadhesive pad to deal with different surface conditions.

\section{Acknowledgments}

The authors acknowledge support from the EPSRC Centre for Innovative Manufacturing in Intelligent Automation, in undertaking this research work under grant reference number EP/IO33467/1. Also, the authors would like to thank Jagpal Singh for his assistance on using the Alicona.

\section{References}

[1] Krape R P 1968 Applications Study of Electroadhesive Devices (NASA Contractor Report: NASA CR-1211) National Aeronautics and Space Administration

[2] Warning R W 1960 Electrostatic force employed to hold workpieces Phys. Forces Electrostatiques Appl. 1 17-27

[3] Beasley G and Hankins W 1971 Development of electroadhesive devices for zero-g intraextravehicular activities AIAA pp 71-853

[4] Jeon J U and Higuchi T 1999 Electrostatic suspension of dielectrics IEEE Trans. Ind. Electron. 45 938-46

[5] Monkman G J, Taylor P M, and Farnworth G J 1989 Principles of electroadhesion in clothing technology Int. J. Clothing Sci. Technol. 1 14-20

[6] Zhang Z, Chestney J A and Sarhadi M 2001 Characterizing an electrostatic gripping device for the automated handling of non-rigid materials Proc. Inst. Mech. Eng. B 215 21-36

[7] Monkman G J 1995 Robot grippers for use with fibrous materials Int. J. Robot. Res. 14 144-51

[8] Nakashim Y T and Higuchi T 2007 Wall climbing mechanisms using electrostatic attraction generated by flexible electrodes Int. Symp. on Micro-NanoMechatronics and Human Science (Nagoya, Japan, 11-14 November 2007) pp 389-94

[9] http://grabitinc.com/

[10] Guo J 2015 A concept selection method for designing climbing robots Key Eng. Mater. $64922-9$

[11] Ruffatto D, Shah J and Spenko M 2014 Improving controllable adhesion on both rough and smooth surfaces with a hybrid electrostatic/gecko-like adhesive J. R. Soc. Interface 11 1-10

[12] Téllez J P D, Krahn J and Menon C 2011 Characterization of electro-adhesives for robotic applications IEEE Int. Conf. on Robotics and Biomimetics (Karon Beach, Phuket, 7-11 December 2011) pp 1867-72

[13] Shim G and Sugai H 2008 Dechuck operation of Coulomb type and Johnsen-Rahbek type of electrostatic chuck used in plasma processing Plasma Fusion Res. 3051

[14] Mao J et al 2014 Modelling and simulation of electrostatic attraction force for climbing robots on the conductive wall material IEEE Int. Conf. on Mechatronics and Automation (Tianjin, China, 3-6 August 2014) pp 987-92

[15] Watanabe T, Kitabayashi T and Nakayama C 1993 Relationship between electrical resistivity and electrostatic force of alumina electrostatic Japan. J. Appl. Phys. 32 864-71

[16] Lossel Y Y, Kochanov E S and Strunskii M G 1981 Calculation of Electrical Capacitance (Moscow: Energoizdat) p 288

[17] Danzl R, Helmli F and Scherer S 2011 Focus variation-a robust technology for high resolution optical 3D surface metrology J. Mech. Eng. 57 245-56

[18] www.digitalsurf.com/en/index.html

[19] Richard L 2011 Optical Measurement of Surface Topography (Berlin: Springer) pp 187-208

[20] Monkman G J 1992 Compliant robotic devices and electroadhesion Robotica 10 183-5

[21] Dhelika R et al 2013 Electrostatic chuck consisting of polymeric electrostatic inductive fibers for handling of objects with rough surfaces Smart Mater. Struct. 22095010 